01

\title{
Анализ электростатических взаимодействий аминокислотных остатков на примере образования димера Nap1-Nap1
}

\author{
(C) Т.В. Кошлан, ${ }^{1}$ К.Г. Куликов ${ }^{2}$ \\ ${ }^{1}$ Санкт-Петербургский государственный университет, \\ 199034 Санкт-Петербург, Россия \\ ${ }^{2}$ Санкт-Петербургский политехнический университет Петра Великого, \\ 195251 Санкт-Петербург, Россия \\ e-mail: kulikov.kirill.g@gmail.com
}

Поступило в Редакцию 3 июня 2019 г.

В окончательной редакции 31 июля 2019 г.

Принято к публикации 31 июля 2019 г.

\begin{abstract}
Представлен новый метод, который позволяет анализировать потенциальную энергию электростатического взаимодействия белковых комплексов при точечных заменах аминокислотных остатков с учетом трехмерной структуры комплекса на примере образования димера Nap1-Nap1. Были разработаны карты потенциальной энергии электростатического взаимодействия попарно взятых аминокислотных остатков участвующих белков. Проведен анализ взаимодействующих белков с учетом трехмерной структуры.
\end{abstract}

Ключевые слова: конформация, гидрофобные взаимодействия, ключевые аминокислотные остатки, кластер.

DOI: $10.21883 /$ JTF.2020.03.48916.229-19

\section{Введение}

В настоящей работе представлен новый метод, но основе которого возможно разрабатывать лекарственные ингибиторы для белков-мишеней, аналогичные ранее разработанным моноклональным антителам таким, как трастузумаб и цетуксимаб. При этом трастузумаб связывается с HER2 рецептором и замедляет дупликацию клеток [1], в свою очередь, цетуксимаб является ингибитором рецептора эпидермального фактора роста (EGFR) [2] и используется для лечения метастатического колоректального рака, метастатического немелкоклеточного рака легких.

Разработанный метод позволит модифицировать существующие известные препараты для лечения патологии рака с целью повышения их сродства к целевым рецепторам, а также учитывать влияние точечных мутаций в белках, которые могут наблюдаться при различных типах опухолей, например, как соматическая мутация гена BRAF в случае меланомы [3]. Изменение даже одного аминокислотного остатка в полипептидной цепи лиганда или рецептора может привести к изменению аффинности всего комплекса „рецептор-лиганд“.

Таким образом, построенная модель позволяет учитывать такие изменения сродства и выбора аминокислоты, состава полипептидных цепей для увеличения аффинности биологического комплекса и повышение селективности связывания.

При этом каждому мутантному патогенному белку мы можем подобрать конкретный ингибитор, принимая во внимание изменения в конформации и перераспределения электростатического потенциала.

Пример такого перераспределения электростатического потенциала при замене аминокислотных остатков в белках, мы разбираем на примере образования димера Nap1-Nap1 ниже.

Представленный в настоящей работе подход позволяет анализировать и идентифицировать наличие гидрофобных кластеров, а также перераспределение потенциальной энергии электростатического взаимодействия при точечных заменах аминокислотных остатков на примере белка Nap1-Nap1.

В настоящей работе разработаны математические алгоритмы, которые позволяют определять ключевые аминокислотные остатки на основе трехмерной структуры путем анализа матрицы потенциальной энергии попарного электростатического взаимодействия между различными сайтами биологического комплекса. При этом замена ключевых а.a. (amino acid) приводит к перераспределению потенциальной энергии попарного электростатического взаимодействия.

В перспективе это позволит модифицировать существующие белки, повышая их аффинность к целевым биологическим молекулам, тем самым приводя к ингибированию или активированию их биологических функций в клетках, что и определяет актуальность представленной работы.

Настоящая работа состоит из несколько частей. В первой части работы описывается структура и функции белка Nap1. Вторая часть посвящена поведению гидрофобных молекул в водном окружении. Третий раздел содержит анализ взаимодействия двух белков на примере образования гомодимера Nap1-Nap1. B четвертой части приводится образование гидрофобного кластера за счет нескольких замен а.а. в белках: E110W,K111W,E112W, E116W одного белка Nap1 и E121W, N122W, Q126W другого белка Nap1 и описание карт белковых взаимо- 
действий на примере димера Nap1-Nap1 с учетом различной природы взаимодействующих аминокислотных остатков (гидрофильных, гидрофобных, заряженных) и величины потенциальной энергии электростатического взаимодействия.

\section{1. Структура и функции белка Nap1}

Белок Nap1 (Nucleosome assembly protein 1) является интегральным компонентом в создании и динамике эукариотического хроматина. Белок Nap1 перемещает гистоны в ядро, учавствует в сборке нуклеосомы и способствует подвижности хроматина [4]. Nap1 широко используется в исследованиях по сборке хроматина, для структурных и функциональных исследований. Помимо его роли в содействии экспериментальному исследованию нуклеосом, высоко консервативный белок Nap1 является одним из наиболее изученных членов группы гистоновых шаперонов.

Рассмотрим более подробно структуру белка гистонового шаперона Nap1. B [5] было обнаружено, что белок Nap1 имеет хорошо структурированный центральный участок, тогда как концевые регионы N-конца и C-конца были в основном неупорядоченные. Центральная область представляет собой участок с 74 по 365 а.а.

Во время процесса димеризации происходит взаимодействие в области домена I с 74 по 160 а.а. между длинными $\alpha 2$-спиралями двух белков в противоположных направлениях. Далее димер стабилизируется петлей $\alpha 2-\alpha 3, \alpha 3$-спиралью и петлей $\alpha 3-\alpha 4$, которые обертываются вокруг $\alpha 2$-спирали партнера по димеризации. Схема димеризации двух белков Nap1-Nap1 показана на рис. 1. Интерфейс димера характеризуется главным образом гидрофобными взаимодействиями по всей длине вовлеченной аминокислотной последовательности $\alpha 2$-спирали.

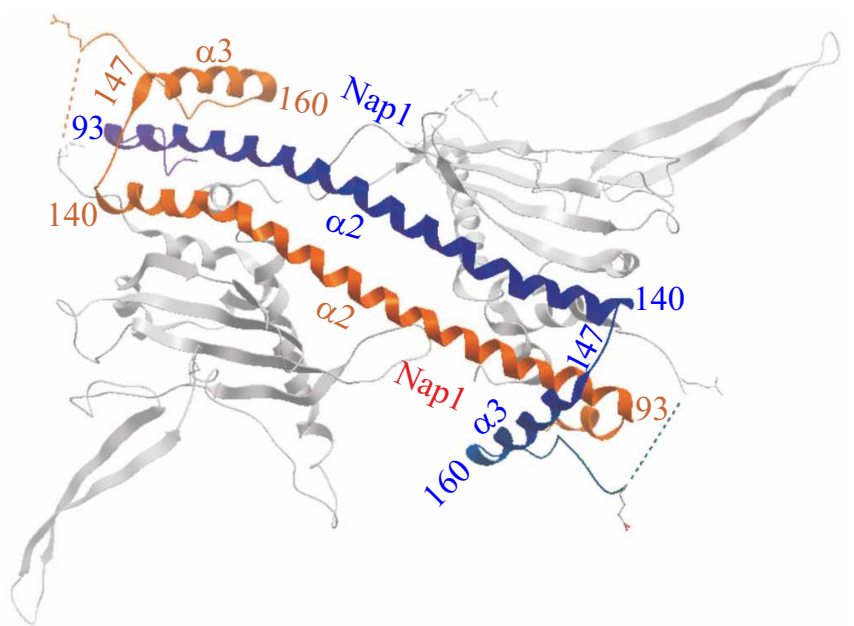

Pис. 1. Диаграмма формирования гомодимера Nap1-Nap1.

\section{2. Взаимодействие гидрофобных молекул в водном окружении}

Особые свойства воды определяют поведение растворенных в ней молекул. Важную роль здесь играет наличие пространственной сетки водородных связей. Отметим, что в жидком состоянии вода образует очень подвижную сетку, в которой водородные связи постоянно переключаются от одной молекулы к другой. Стремление молекул воды к образованию оптимальной сетки приводит к феномену „гидрофобное взаимодействие“, которое трактуется как „сила“, заставляющая молекулы двигаться друг к другу [6]. Природа этой силы иная, чем у кулоновских или ван-дер-ваальсовских сил. Это взаимодействие не является прямым, а действует через реорганизацию структуры окружающей воды, сетки водородных связей. Вода стремится сформировать оптимальную сетку водородных связей, учитывающую присутствие гидрофобных молекул, что принуждает их к сближению и тем самым к формированию кластеров и клатратных структур.

Рассмотрим этот процесс более подробно. Мы предполагаем, что положительные значения потенциальной энергии попарного электростатического взаимодействия между гидрофобными аминокислотными остатками говорят нам об отталкивающей природе на малых расстояниях (в нашем случае). Однако при помещении их в водное окружение, которое характеризуется высокой степенью упорядоченного взаимодействия между молекулами воды, будет приводить к образованию гидрофобных кластеров.

Отметим, что молекулы воды имеют тенденцию к сильному взаимодействию между собой по причине неравномерного распределения электронной плотности между атомом кислорода и двумя атомами водорода. Характер образования молекулы воды таков, что электронная плотность вокруг атома кислорода гораздо больше, чем в области атомов водорода, что, в свою очередь, приводит к образованию диполя, концы которого несут разный по знаку заряд. Наличие такого диполя молекулы воды приводит к сильному взаимодействию с другим диполем молекулы воды: область атома кислорода несет отрицательный заряд, а области атомов водорода несут положительный заряд. В результате атом кислорода одной молекулы притягивает атом водорода другой молекулы воды, образуя высокоупорядоченную структуру диполей молекул воды. Мы предполагаем, что если поместить в такую упорядоченную структуру диполей воды гидрофобные молекулы, между которыми нет таких сильных диполь-дипольных взаимодействий, но существуют дисперсионные взаимодействия, то они будут ее нарушать.

Отметим, что дисперсионные взаимодействия возникают между атомами вследствие флуктуации электронной плотности вокруг ядра, что приводит к появлению мгновенного дипольного момента у атомов 
$a$



$b$

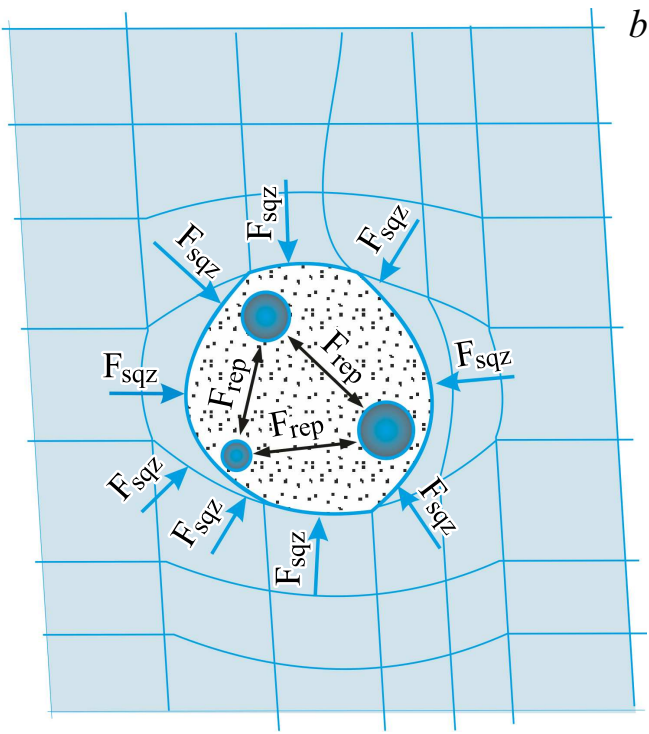

Рис. 2. Поведение гидрофобных молекул в водном окружении с указанием действующих сил, $F_{\text {rep }}-$ сила электростатического взаимодействия, $F_{\mathrm{sqz}}-$ сила сжатия (сдавливания).

и взаимодействию соответствующих диполей. Дисперсионное взаимодействие имеет место между любыми атомными группировками, но оно больше в тех случаях, когда поляризуемость атомов выше. Также в белках дисперсионные силы играют важную роль во взаимодействии аминокислотных остатков, имеющих гидрофобные (неполярные) боковые радикалы, таких как, например, валин, лейцин, триптофан, фенилаланин, тирозин. Энергия дисперсионных взаимодействий обратно пропорциональна шестой степени расстояния между взаимодействующими атомами. Это значит, что дисперсионные силы играют существенную роль лишь на очень коротких расстояниях.

На рис. 2, а представлены упорядоченные ионы воды, которые в процессе упорядочивания развивают силу сжатия $\left(F_{\mathrm{sqz}}\right)$, которая действует на гидрофобные молекулы, которые не участвуют в образовании ионных связей с ионами воды. На рис. $2, b$ схематично представлена водородная сетка, внутрь которой помещены гидрофобные молекулы, между гидрофобными молекулами, зажатыми ионами воды, действуют силы электростатического взаимодействия $\left(F_{\text {rep }}\right)$.

Молекулы воды имеют тенденцию к сильному взаимодействию и будут по всему объему соприкосновения с гидрофобными молекулами „толкать“ их, чтобы они заняли минимальный объем в водном окружении и не препятствовали образованию упорядоченной структуры водных диполей. Таким образом, на гидрофобные молекулы, помещенные в водное окружение, с внешней стороны действует сила сжатия молекул воды (скивизинг) в процессе упорядочивания, который способствует образованию конгломератов из гидрофобных молекул, а с другой стороны на структуры (между гидрофобными молекулами) дей- ствует сила электростатического взаимодействия (положительная по значению), которая приводит к отталкиванию гидрофобных молекул друг от друга на малых расстояниях (рис. 2). Так как на малых расстояниях начинают сказываться силы отталкивания между атомами, которые связаны с эффектами отталкивания электронных облаков при их взаимопроникновении [7].

Данное предположение мы объясняем тем, что молекула имеет как положительный заряд, так и отрицательный, оба эти заряды распределены по молекуле неравномерно, в результате чего какая-то сторона молекулы чуть больше заряжена положительно, другая сторона - чуть больше заряжена отрицательно. Кроме того, значительная часть отрицательного заряда распределена по „внешней“ электронной оболочке, а положительный сосредоточен внутри в ядрах атомов входящих в состав молекул. Сначала это приводит к тому, что молекулы поворачиваются друг к другу разноименно заряженными сторонами (плюсом к минусу). Потом, из-за того что электрическое поле вокруг заряда неравномерное, т.е. имеет градиент, в смысле ослабляется с расстоянием (пропорционально квадрату расстояния), на сравнительно больших расстояниях получается, что сила притяжения между повернутыми друг к другу разноименно заряженными частями чуть-чуть больше силы отталкивания между одноименно заряженными сторонами (хоть и заряды равны, но из-за поворота ориентации расстояния разные, и силы разные), но когда молекулы приблизятся настолько близко, что их электронные оболочки окажутся очень близко друг к другу (в нашем случае), получится, что сила отталкивания между одноименно заряженными электронными оболочками превысит притяжение между 


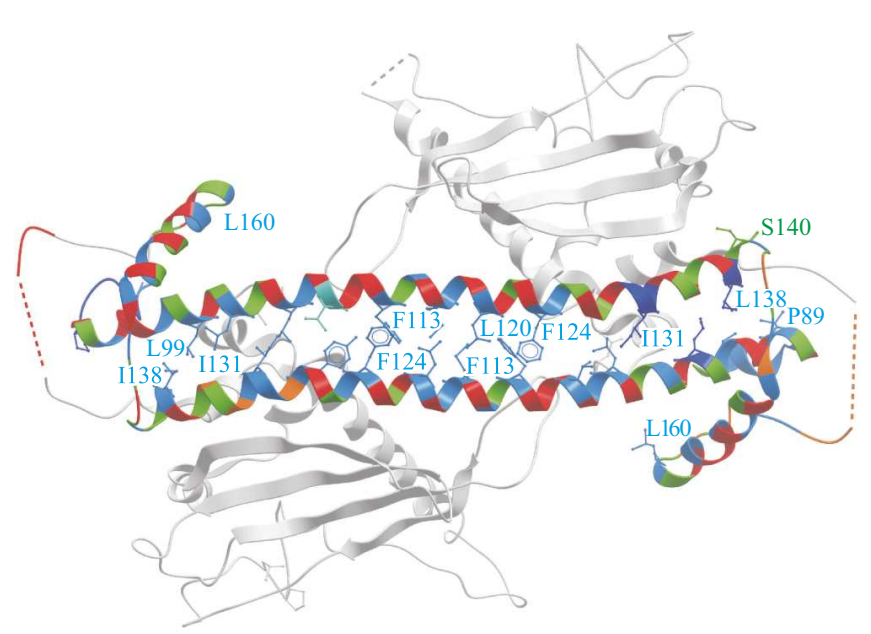

Pис. 3. Структура гомодимера Nap1-Nap1 с указанием гидрофобных, гидрофильных, заряженных а.а.

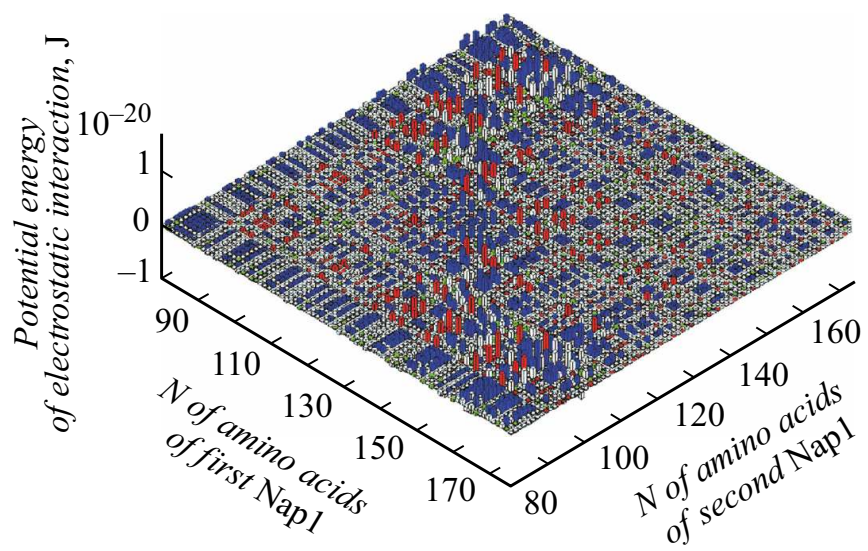

Pис. 4. График трехмерного представления энергии попарного электростатического взаимодействия (ПЭЭВ) с учетом природы взаимодействующих а.а., высота бара соответствует значению ПЭЭВ а.а.

ядрами одной молекулы и электронными оболочками другой, и молекулы оттолкнуться друг от друга на таком расстоянии. При этом силы сжатия со стороны диполей молекул воды гораздо больше сил отталкивания между гидрофобными молекулами, что приводит к возможности образования димерных белковых комплексов между белками, как в нашем случае димера Nap1-Nap1, который образован за счет гидрофобных взаимодействий [5].

Отметим, что на основе разработанной нами модели появилась возможность количественной оценки гидрофобного эффекта методами компьютерного моделирования: рассчитать значение потенциальной энергии попарного электростатического взаимодействия между каждой парой гидрофобных аминокислотных остатков двух полипептидных цепей белков с учетом конформации.

\section{3. Анализ взаимодействия двух белков на примере образования гомодимера Nap1-Nap1}

В разд. 3 будут представлены результаты численного моделирования связывания двух белков Nap1-Nap1 в виде трехмерного представления электростатического попарного взаимодействия с указанием гидрофильных, гидрофобных взаимодействий, а также взаимодействий заряженных аминокислотных остатков двух одинаковых белков Nap1 и Nap1.

Для получения карты белковых взаимодействий (Nap1 и Nap1) была рассчитана матрица потенциальной энергии электростатического взаимодействия двух белков Nap1 и Nap1. При этом данные о трехмерной структуре были взяты из базы PDB под номером: 5G2E [8]. B [8] первый белок Nap1 начинается с 82 a.a. [S] и заканчивается 365 a.a. [L], второй белок Nap1 начинается c 83 a.a. [G] и заканчивается 365 a.a [L]. Эти данные представляют собой хорошо упорядоченные структуры, слабоупорядоченные последовательности со стороны N-
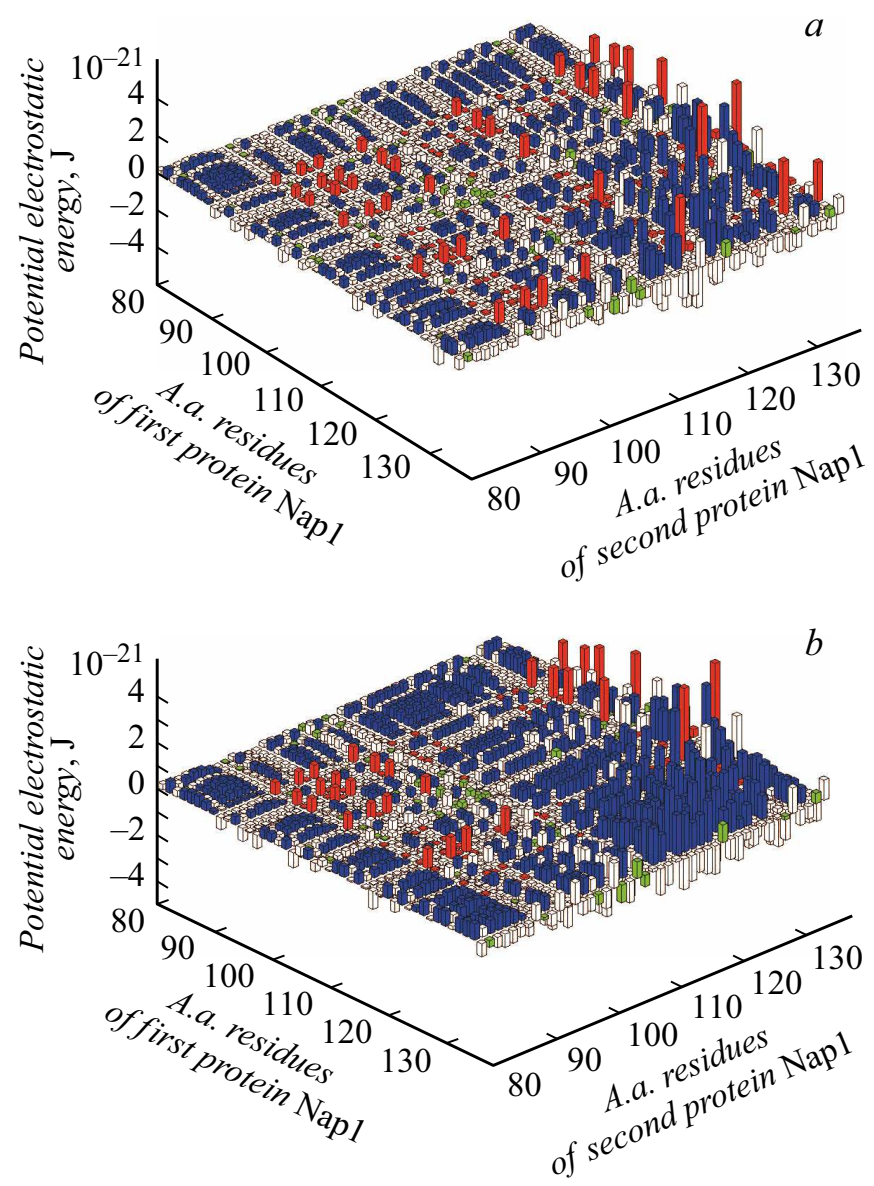

Рис. 5. Трехмерное представление потенциальной энергии попарного электростатического взаимодействия двух белков Nap1 и Nap1 при образовании гомодимера в случае белков дикого типа и в случае образования гидрофобного кластера. Высота бара отвечает значению потенциальной энергии попарного электростатического взаимодействия. 
Таблица 1.

\begin{tabular}{|c|c|c|c|c|c|c|c|}
\hline \multirow{3}{*}{$\begin{array}{c}\text { А.а. } \\
\text { второго белка Nap1 }\end{array}$} & \multicolumn{7}{|c|}{$\begin{array}{c}\text { Энергия электростатического взаимодействия } \\
\text { между а.а. белка Nap1, J }\end{array}$} \\
\hline & \multicolumn{7}{|c|}{ Дикий тип } \\
\hline & GLU110 & LYS111 & GLU112 & PHR113 & GLU114 & VAL115 & GLU116 \\
\hline GLU121 & $2.64 \cdot 10^{-23}$ & $2.46 \cdot 10^{-22}$ & $2.34 \cdot 10^{-23}$ & $-3.3 \cdot 10^{-22}$ & $1.88 \cdot 10^{-22}$ & $-1.8 \cdot 10^{-22}$ & $2.78 \cdot 10^{-23}$ \\
\hline ASN122 & $-4 \cdot 10^{-23}$ & $-3.9 \cdot 10^{-22}$ & $-4 \cdot 10^{-23}$ & $4.1 \cdot 10^{-22}$ & $-2.5 \cdot 10^{-22}$ & $2.75 \cdot 10^{-22}$ & $-4.5 \cdot 10^{-23}$ \\
\hline LYS123 & $2.87 \cdot 10^{-22}$ & $3.06 \cdot 10^{-21}$ & $3.74 \cdot 10^{-22}$ & $-3.2 \cdot 10^{-21}$ & $1.86 \cdot 10^{-21}$ & $-2.3 \cdot 10^{-21}$ & $4.41 \cdot 10^{-22}$ \\
\hline PHE124 & $-2.8 \cdot 10^{-22}$ & $-2.6 \cdot 10^{-21}$ & $-3.3 \cdot 10^{-22}$ & $3.39 \cdot 10^{-21}$ & $-1.6 \cdot 10^{-21}$ & $1.84 \cdot 10^{-21}$ & $-3.1 \cdot 10^{-22}$ \\
\hline LEU125 & $-2.1 \cdot 10^{-22}$ & $-1.8 \cdot 10^{-21}$ & $-1.7 \cdot 10^{-22}$ & $1.77 \cdot 10^{-21}$ & $-1.1 \cdot 10^{-21}$ & $1.1 \cdot 10^{-21}$ & $-1.6 \cdot 10^{-22}$ \\
\hline GLN126 & $1.44 \cdot 10^{-22}$ & $1.38 \cdot 10^{-21}$ & $1.39 \cdot 10^{-22}$ & $-1.2 \cdot 10^{-21}$ & $7.68 \cdot 10^{-22}$ & $-8.8 \cdot 10^{-22}$ & $1.36 \cdot 10^{-22}$ \\
\hline \multirow[t]{3}{*}{ LYS127 } & $3.73 \cdot 10^{-22}$ & $3.59 \cdot 10^{-21}$ & $4 \cdot 10^{-22}$ & $-3 \cdot 10^{-21}$ & $1.78 \cdot 10^{-21}$ & $-2.1 \cdot 10^{-21}$ & $3.21 \cdot 10^{-22}$ \\
\hline & \multicolumn{7}{|c|}{ Мутации } \\
\hline & TRP110 & TRP111 & TRP112 & PHE113 & GLN114 & VAL115 & TRP116 \\
\hline TRP121 & $1.59 \cdot 10^{-21}$ & $1.19 \cdot 10^{-21}$ & $1.41 \cdot 10^{-21}$ & $2.55 \cdot 10^{-21}$ & $-1.5 \cdot 10^{-21}$ & $1.38 \cdot 10^{-21}$ & $1.67 \cdot 10^{-21}$ \\
\hline ASN122 & $3.08 \cdot 10^{-22}$ & $2.43 \cdot 10^{-22}$ & $3.06 \cdot 10^{-22}$ & $4.1 \cdot 10^{-22}$ & $-2.5 \cdot 10^{-22}$ & $2.75 \cdot 10^{-22}$ & $3.5 \cdot 10^{-22}$ \\
\hline TRP123 & $1.39 \cdot 10^{-21}$ & $1.2 \cdot 10^{-21}$ & $1.81 \cdot 10^{-21}$ & $2.02 \cdot 10^{-21}$ & $-1.2 \cdot 10^{-21}$ & $1.46 \cdot 10^{-21}$ & $2.14 \cdot 10^{-21}$ \\
\hline PHE124 & $2.14 \cdot 10^{-21}$ & $1.64 \cdot 10^{-21}$ & $2.55 \cdot 10^{-21}$ & $3.39 \cdot 10^{-21}$ & $-1.6 \cdot 10^{-21}$ & $1.84 \cdot 10^{-21}$ & $2.42 \cdot 10^{-21}$ \\
\hline LEU125 & $1.63 \cdot 10^{-21}$ & $1.12 \cdot 10^{-21}$ & $1.32 \cdot 10^{-21}$ & $1.77 \cdot 10^{-21}$ & $-1.1 \cdot 10^{-21}$ & $1.1 \cdot 10^{-21}$ & $1.27 \cdot 10^{-21}$ \\
\hline GLN126 & $-1.1 \cdot 10^{-21}$ & $-8.6 \cdot 10^{-22}$ & $-1.1 \cdot 10^{-21}$ & $-1.2 \cdot 10^{-21}$ & $7.68 \cdot 10^{-22}$ & $-8.8 \cdot 10^{-22}$ & $-1.1 \cdot 10^{-21}$ \\
\hline TRP127 & $1.81 \cdot 10^{-21}$ & $1.41 \cdot 10^{-21}$ & $1.94 \cdot 10^{-21}$ & $1.87 \cdot 10^{-21}$ & $-1.1 \cdot 10^{-21}$ & $1.34 \cdot 10^{-21}$ & $1.56 \cdot 10^{-21}$ \\
\hline
\end{tabular}

и С-конца [1-81 а.а] и [367-417 а.а.] отсутствуют в базе данных и не будут принимать участие при расчете матрицы потенциальной энергии электростатического взаимодействия двух белков, так же отсутствуют участки с 172 по 180 а.а. и с 285 по 307 а.а. Отметим, что мы присвоили порядковые номера присутствующим аминокислотным остаткам двух белков, начиная с № 82 для первого белка Nap1 и с № 83 для второго белка Nap1.

На рис. 3 представлен гомодимер Nap1-Nap1, в цвете обозначен Domain 1 каждого из белков, который принимает активное участие в образование димера. В расчетах будет присутствовать полипептидная цепь двух белков, представленная в [8]. На рис. 3 отмечены аминокислотные остатки, обращенные в сторону полипептидной последовательности второго белка, как видно из представленного рисунка, большинство аминокислотных остатков в данном случае являются гидрофобными. Образование димера осуществляется за счет соединения полипептидных цепей двух белков в противоположном направлении. Так же из рисунка следует, что образование димера Nap1-Nap1 осуществляется в основном гидрофобными аминокислотными остатками.

Выполним расчет потенциальной энергии электростатического взаимодействия двух белков Nap1 и Nap1.

Так, на рис. 4 показана карта потенциальной энергии электростатического взаимодействия, где мы видим различные цветовые бары, высота которых будет отвечать конкретному значению энергии, которая была вычислена между каждой парой аминокислотных остатков двух полипептидных цепей. На представленном графике наблюдаются диагональные пики (возмущения). Полученное графическое представление позволило проанализировать эффект точечных замен аминокислотных остатков в полипептидных цепях белков (см. разд. 4.1).

\section{4. Образование гидрофобного кластера за счет нескольких замен а.а. в белках: E110W, K111W, E112W, E116W одного белка Nap1 и E121W, N122W, Q126W другого белка Nap1}

Для удобства и наглядности представления, мы будем показывать часть матрицы потенциальной энергии попарного электростатического взаимодействия, фрагмент с 82 по 122 a.a.

На рис. 5, а представлено графическое отображение матрицы потенциальной энергии взаимодействия с 82 по 120 a.a. белка Nap1-Nap1 дикого типа. На графике 5,b показано образование белка Nap1-Nap1 при замене аминокислотных остатков Е110, K111, E112, Е116 одного белка Nap1 и E121, N122, Q126 другого белка Nap1 на гидрофобный триптофан (W).

Как видно из приведенного рис. 5, наличие гидрофобного кластера привело к более равномерному распределению потенциальной энергии взаимодействия в положительных значениях по сравнению со взаимодействием белков дикого типа (wt). 
Таблица 2.

\begin{tabular}{|c|c|c|}
\hline \multirow{3}{*}{$\begin{array}{l}\text { А.а. } \\
\text { второго белка }\end{array}$} & \multicolumn{2}{|c|}{$\begin{array}{c}\text { Энергия электростатического взаимодействия } \\
\text { между а.а. белка Nap1, J }\end{array}$} \\
\hline & Дикий тип & Мутации \\
\hline & THR101 & ALA101 \\
\hline $\begin{array}{c}\text { GLU133 } \\
\text { GLN134 } \\
\text { ARG135 } \\
\text { SER136 } \\
\text { AEG137 } \\
\text { ILE138 } \\
\text { ILE139 } \\
\text { SER140 } \\
\text { GLY141 } \\
\text { GLN142 } \\
\text { GLU143 } \\
\text { GLN144 } \\
\text { PRO145 } \\
\text { LYS146 } \\
\text { PRO147 } \\
\text { GLU148 } \\
\text { GLN149 } \\
\text { ILE150 } \\
\text { ALA151 } \\
\text { LYS152 } \\
\text { GLY153 } \\
\text { GLN154 } \\
\text { GLU155 }\end{array}$ & $\begin{array}{r}-1.527 \cdot 10^{-22} \\
-1.482 \cdot 10^{-21} \\
-2.234 \cdot 10^{-21} \\
7.854 \cdot 10^{-22} \\
-1.639 \cdot 10^{-21} \\
1.968 \cdot 10^{-21} \\
1.580 \cdot 10^{-21} \\
6349 \cdot 10^{-22} \\
1.462 \cdot 10^{-21} \\
-8.651 \cdot 10^{-22} \\
-1.681 \cdot 10^{-22} \\
-1.068 \cdot 10^{-21} \\
6.058 \cdot 10^{-22} \\
-2.755 \cdot 10^{-21} \\
5.555 \cdot 10^{-22} \\
-2.325 \cdot 10^{-22} \\
-2.611 \cdot 10^{-21} \\
2.505 \cdot 10^{-21} \\
3.789 \cdot 10^{-21} \\
-4.619 \cdot 10^{-21} \\
3.841 \cdot 10^{-21} \\
-1.432 \cdot 10^{-21} \\
-2.280 \cdot 10^{-22}\end{array}$ & $\begin{array}{r}-2.945 \cdot 10^{-22} \\
-2.858 \cdot 10^{-21} \\
-4.307 \cdot 10^{-21} \\
1.514 \cdot 10^{-21} \\
-3.161 \cdot 10^{-21} \\
3.795 \cdot 10^{-21} \\
3.04 \cdot 10^{-21} \\
1.22 \cdot 10^{-21} \\
2.818 \cdot 10^{-21} \\
-1.668 \cdot 10^{-21} \\
-3.242 \cdot 10^{-22} \\
-2.059 \cdot 10^{-21} \\
1.168 \cdot 10^{-21} \\
-5.312 \cdot 10^{-21} \\
1.071 \cdot 10^{-21} \\
-4.484 \cdot 10^{-22} \\
-5.034 \cdot 10^{-21} \\
4.831 \cdot 10^{-21} \\
7.307 \cdot 10^{-21} \\
-8.907 \cdot 10^{-21} \\
7.406 \cdot 10^{-21} \\
-2.761 \cdot 10^{-21} \\
-4.396 \cdot 10^{-22}\end{array}$ \\
\hline
\end{tabular}

Проанализируем данные табл. 1 в выбранной области полипептидной последовательности с 110 по 116а.а.

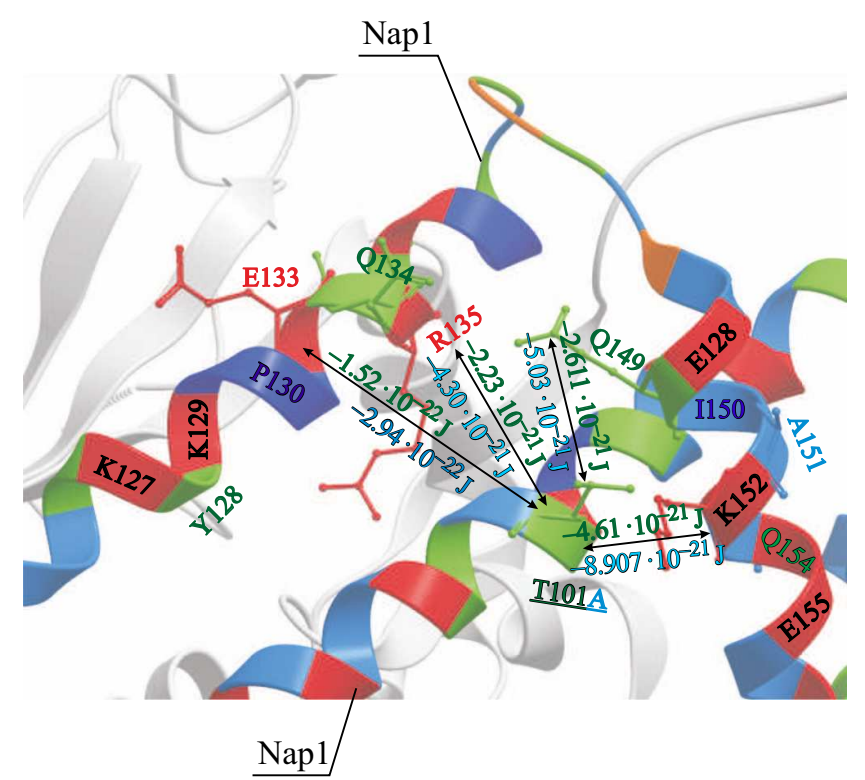

Рис. 6. Участок полипептидных цепей белков Nap1 и Nap1 c указанием ключевых а.а. при замене T101A, а также с указанием изменения потенциальной энергии электростатического взаимодействия до и после замены. первого белка Nap1 и с 121 по 127 а.а. второго белка Nap1 с мутациями и без мутаций.

Аминокислотные остатки двух полипептидных цепей двух белков Nap1, которые мы заменяли на гидрофобный триптофан подчеркнуты. При замене аминокислотных остатков (6 заряженных а.а. и 1 гидрофильный а.а.) на гидрофобные аминокислотные остатки наблюдается существенное преобладание потенциальной энергии попарного электростатического взаимодействия в положительной области „высоких“ значений (двадцать первая степень). Такое поведение гидрофобных аминокислотных остатков в водном окружении можно интерпретировать, как „вынужденное притяжение“. При этом мы предполагаем, что именно водное окружение „заставляет“ гидрофобные аминокислотные остатки сближаться (см. разд. 2).

\section{1. Замена гидрофильного а.а. T101A на гидрофобный аминокислотный остаток}

При взаимодействии белков дикого типа Nap1-Nap1 Т101 (треонин 101) характеризуется достаточно большим отрицательным значением потенциальной энергии с К152 по сравнению со взаимодействиями остальных а.а., таким образом, была выполнена замена гидрофильного треонин на гидрофобный аланин (А) и пе- 
ресчитана потенциальная энергия электростатического взаимодействия для такой замены.

Приведем часть полученной матрицы в области ключевых аминокислотных остатков двух взаимодействующих белков до и после замены Т101А.

Как видно из табл. 2, при замене Т101А произошло увеличение абсолютного значения потенциальной энергии взаимодействия. Так же появились значения потенциальной энергии в положительной области значений, отвечающие взаимодействию гидрофобных а.а., что мы рассматриваем, как „притягивание“ в водной среде.

На рис. 6 представлен участок двух полипептидных цепей белка Nap1 и Nap1 с указанием ключевых а.a., а так же указанием изменения значений потенциальной энергии электростатического взаимодействия до и после замены Т101А

\section{Выводы}

В статье разработан метод, позволяющий модифицировать существующие белки путем введения точечных замен ключевых а.а., которые идентифицированы предложенным подходом.

В результате выполненных численных расчетов на основе построенной физической модели были получены следующие результаты:

1. Разработаны карты потенциальной энергии электростатического взаимодействия попарно взятых а.а. участвующих белков на примере Nap1-Nap1, которые позволяют наглядно увидеть характер образования белкового комплекса, идентифицировать максимумы и минимумы потенциальной энергии между а.а. двух белков, определять ключевые а.а., на которые приходятся максимальные значения потенциальной энергии. Особое внимание уделено природе взаимодействующих а.а. (гидрофобные, гидрофильные, заряженные). Проведен анализ взаимодействующих белков с учетом трехмерной структуры, где были обозначены участки с наибольшим значением потенциальной энергии электростатического взаимодействия.

2. На основе разработанной модели появилась возможность количественной оценки гидрофобного эффекта методами компьютерного моделирования: рассчитать значение потенциальной энергии электростатического взаимодействия между каждой парой гидрофобных а.а. двух полипептидных цепей белков с учетом конформации.

Проведен научно-обоснованный расчет эффектов, связанных с заменой ключевых а.а., замена которых может влиять на аффинность взаимодействующих белков, а это, в свою очередь, обусловливает прохождение биохимических реакций в клетке.

\section{Конфликт интересов}

Авторы заявляют, что у них нет конфликта интересов.

\section{Список литературы}

[1] Valabrega G., Montemurro F., Aglietta M. // Ann Oncol. 2007. Vol. 18. N 6. P. 977-984.

[2] Harding J., Burtness B. // Drugs Today (Barc). 2005. Vol. 41. N 2. P. $107-127$.

[3] Рациональная фармакотерапия в онкологии / Под. ред. М.И. Давыдова, В.А. Горбуновой. М.: ЛитТерра, 2017. 880 р.

[4] Zlatanova J., Seebart C., Tomschik M. // FASEB J. 2007. Vol. 21. N 7. P. 1294-1310.

[5] Park Y.J., Luger K. // Proc. Natl. Acad. Sci. USA. 2006. Vol. 103. N 5. P. $1248-1253$.

[6] Wypych G. Handbook of solvents. ChemTec Publishing, 2001. $1680 \mathrm{p}$.

[7] Рубин А.Б. Биофизика. Т. 1. Теоретическая биофизика. М.: МГУ, 1999. $448 \mathrm{c.}$

[8] Protein data bank-URL:https://www.rcsb.org/(дата обращения 17.04.2019) 